\title{
BMJ Open Using nasal povidone-iodine to prevent bloodstream infections and transmission of Staphylococcus aureus among haemodialysis patients: a stepped-wedge cluster randomised control trial protocol
}

\author{
Ana-Monica Racila (D) , ${ }^{1}$ Amy M J O'Shea, ${ }^{1}$ Rajeshwari Nair, ${ }^{1}$ Kimberly Dukes, ${ }^{1}$ \\ Loreen A Herwaldt, ${ }^{1}$ Linda Boyken, ${ }^{1}$ Daniel Diekema, ${ }^{1}$ Melissa A Ward, ${ }^{1}$ \\ Jason Cobb, ${ }^{2}$ Jesse Jacob, ${ }^{3}$ David Pegues, ${ }^{4}$ Susan Bleasdale, ${ }^{5}$ Anitha Vijayan, ${ }^{6}$ \\ Anubha Mutneja, ${ }^{6}$ Mony Fraer, ${ }^{1}$ Debra O'Connell-Moore, ${ }^{7}$ Pam Tolomeo, ${ }^{8}$ \\ Minerva Mendez, ${ }^{9}$ Erin Jaworski, ${ }^{10}$ Marin L Schweizer ${ }^{1}$
}

To cite: Racila A-M,

O'Shea AMJ, Nair R, et al. Using nasal povidone-iodine to prevent bloodstream infections and transmission of Staphylococcus aureus among haemodialysis patients: a stepped-wedge cluster randomised control trial protocol. BMJ Open 2021;11:e048830. doi:10.1136/ bmjopen-2021-048830

- Prepublication history for this paper is available online. To view these files, please visit the journal online (http://dx.doi. org/10.1136/bmjopen-2021048830).

Received 14 January 2021 Accepted 28 October 2021

Check for updates

(c) Author(s) (or their employer(s)) 2021. Re-use permitted under CC BY-NC. No commercial re-use. See rights and permissions. Published by BMJ.

For numbered affiliations see end of article.

Correspondence to

Dr Ana-Monica Racila;

ana-monica-racila@uiowa.edu

\section{ABSTRACT}

Introduction Approximately $38 \%$ of haemodialysis patients carry Staphylococcus aureus in their noses, and carriers have a nearly four-fold increased risk of $S$. aureus access-related bloodstream infections (BSIs) compared with non-carriers. Our objective is to determine the clinical efficacy and effectiveness of a novel intervention using nasal povidone-iodine (PVI) to prevent BSIs among patients in haemodialysis units. We will survey patients and conduct qualitative interviews with healthcare workers to identify barriers and facilitators to implementing the intervention.

Methods and analysis We will perform an open-label, stepped-wedge cluster randomised trial to assess the effectiveness of nasal PVI compared with standard care. Sixteen outpatient haemodialysis units will participate in the study. The 3-year trial period will be divided into a 4-month baseline period and eight additional 4-month time blocks. The primary outcome of the study will be $S$. aureus BSI, defined as a $S$. aureus positive blood culture collected in the outpatient setting or within one calendar day after a hospital admission. The study team will evaluate characteristics of individual patients and the clusters by exposure status (control or intervention) to assess the balance between groups, and calculate descriptive statistics such as average responses separately for control and intervention survey questions.

Ethics and dissemination This study has received IRB approval from all study sites. A Data Safety and Monitoring Board will monitor this multicentre clinical trial. We will present our results at international meetings. The study team will publish findings in peer-reviewed journals and make each accepted peer-reviewed manuscript publicly available.

Trial registration number NCT04210505.

\section{INTRODUCTION}

Patients on chronic haemodialysis are an ideal target population in whom to implement
Strengths and limitations of this study

Novel intervention targets a modifiable risk factor for Staphylococcus aureus bloodstream infections in patients on haemodialysis.

- Stepped-wedge cluster randomised trial design allows units to serve as their own controls and as a control for other units, thus limiting selection bias and imbalance among the intervention and control units.

- Nasal povidone-iodine suppresses bacteria for only 12-24hours and must be reapplied before each procedure.

interventions to decrease rates of Staphylococcus aureus infections. More than 400000 patients received haemodialysis in 2018, and the majority of these patients received in-centre haemodialysis. ${ }^{1}$ Between 2005 and 2008, 43\% of patients on haemodialysis tracked in the US Renal Data System were hospitalised for infection-related diagnoses. ${ }^{2}$ Approximately $30 \%$ of bloodstream infections (BSIs) among patients on haemodialysis are caused by $S$. aureus ${ }^{34}$ and these infections cause considerable morbidity ${ }^{5-8}$ and mortality. ${ }^{79}$

Several factors increase the risk for $S$. aureus infections among patients on haemodialysis. First, a substantial proportion (38\%) of these patients carry $S$. aureus in their noses, and carriers have a nearly four-fold increased risk of $S$. aureus access-related BSI compared with non-carriers. ${ }^{10}$ Second, these patients have impaired immune function, which makes them more susceptible to infection. ${ }^{11}$ Third, S. aureus can colonise the skin on patients' vascular access sites (arteriovenous grafts or fistulae) and this organism can be introduced 
into bloodstream when the skin is punctured or dialysis catheters are accessed. ${ }^{12-15}$ Fourth, the S. aureus colonising one patient can be transmitted to other patients in the same haemodialysis unit. We previously found that $87 \%$ of patients on dialysis who carried S. aureus in their noses and on their hands carried the same strains at both sites, suggesting transmission from the patients' noses to their skin. ${ }^{16}$ The $S$. aureus strains can then be transmitted from patient-to-patient in a haemodialysis unit via direct contact between patients and healthcare workers' (HCWs) hands and indirectly by contaminated furniture and equipment. ${ }^{17}$ Unlike many other risk factors for BSI in this patient population (eg, comorbidities), S. aureus nasal carriage is modifiable and thus our intervention could substantially benefit this population. ${ }^{2} 318$

To date, studies that evaluated nasal decolonisation of patients on haemodialysis assessed the efficacy of intranasal mupirocin ointment for decolonisation and infection prevention. ${ }^{19}$ However, few dialysis centres have included mupirocin decolonisation as a standard practice due to implementation barriers such as concern for mupirocin resistance and complicated protocols. ${ }^{19-22}$ Povidone-iodine (PVI) has been used as an antiseptic in the healthcare setting for decades and PVI resistance has not been found. ${ }^{23}{ }^{24}$ Thus, nasal PVI can be given to all patients who are not allergic to iodine regardless of their colonisation status. $5 \%$ PVI ( $\mathrm{w} / \mathrm{w}(0.5 \%$ available iodine) USP) is available under the US Food and Drug Administration (FDA) Final Rule. ${ }^{25}$ Our objective is to perform a multicentre stepped-wedge cluster randomised trial (CRT) to determine the clinical efficacy and effectiveness of a novel intervention using nasal PVI to prevent BSIs among patients on hamodialysis. We will survey patients and conduct qualitative interviews with HCWs to identify barriers and facilitators to implementing the intervention.

\section{Objective 1}

Conduct a multicentre, stepped-wedge CRT to determine whether nasal PVI decolonisation reduces infections among patients on haemodialysis.

\section{Objective 2}

Survey patients to assess their satisfaction with nasal PVI decolonisation, assess PVI's role in patient activation around their own health before and after PVI use, and identify barriers and facilitators to implementation.

\section{Objective 3}

Examine HCW satisfaction with implementation of nasal PVI decolonisation and assess barriers and facilitators to the process via qualitative interviews and site visits.

\section{METHODS AND ANALYSIS}

\section{Study design}

We will perform an open-label, stepped-wedge CRT to assess the effectiveness of nasal PVI compared with standard care. Our objectives are to evaluate whether using intranasal PVI will reduce rates of $S$. aureus BSI among patients on haemodialysis, to qualitatively evaluate the implementation of this intervention, and to measure HCW and patient satisfaction with PVI. We will randomly assign when haemodialysis units (clusters) will cross over from the control group to the intervention group such that all units will eventually receive the intervention. ${ }^{26}$ The control group will consist of standard care as regulated by US Centers for Medicare and Medicaid Services (CMS). We will include new patients who begin haemodialysis and stop patient follow-up when a patient is no longer on haemodialysis (eg, recovery of kidney function, kidney transplantation or death). ${ }^{2}$

Sixteen outpatient haemodialysis units will participate in the study. The 3-year trial period will be divided into a 4-month baseline period and eight additional 4-month time blocks (table 1). All units will begin in the control condition (C; no intervention). Two units (a unit pair) will be added to the intervention (I) in a stepwise fashion at the beginning of the eight additional time blocks. ${ }^{26}$

\section{Study setting and participants}

The proposed research will be performed at outpatient haemodialysis units affiliated with five US academic medical centres in the Southeast, Midwest and Northeast. This multicentre study of geographically diverse hospital systems and their patient populations will improve the external validity of our study. We have confirmed that none of the study sites currently perform nasal decolonisation.

We will enrol patients if they are 18 years or older and receiving outpatient chronic haemodialysis (three sessions a week). We will exclude patients receiving peritoneal dialysis or home haemodialysis, patients with documented or verbalised sensitivity or allergy to iodine or iodine-based contrast, patients with known pregnancy, and patients on treatment for bacterial infection. We will enroll adult HCWs working at any of the 16 haemodialysis units who are willing to participate in the semistructured interviews.

\section{Screening and recruitment}

Research team members at each dialysis centre will identify patients that meet inclusion criteria and will discuss the study with patients during a haemodialysis session, while ensuring that patient care is not delayed or disrupted. This study was approved with a waiver of signed consent, as the study is deemed low-risk and patients may have trouble writing while receiving haemodialysis. Thus, patients who verbally agree to the informed consent will be included in the study.

Two research team anthropologists will schedule and conduct semistructured interviews with 5-10 HCW at haemodialysis units across the five centres to examine the contextual factors that influence adoption of nasal PVI decolonisation. The anthropologists will ask each unit's medical director for permission to interview staff and for the names of potential interviewees. The anthropologists will invite potential interviewees-the nurse manager, a physician, nurses, nursing assistants or technicians-to participate. 


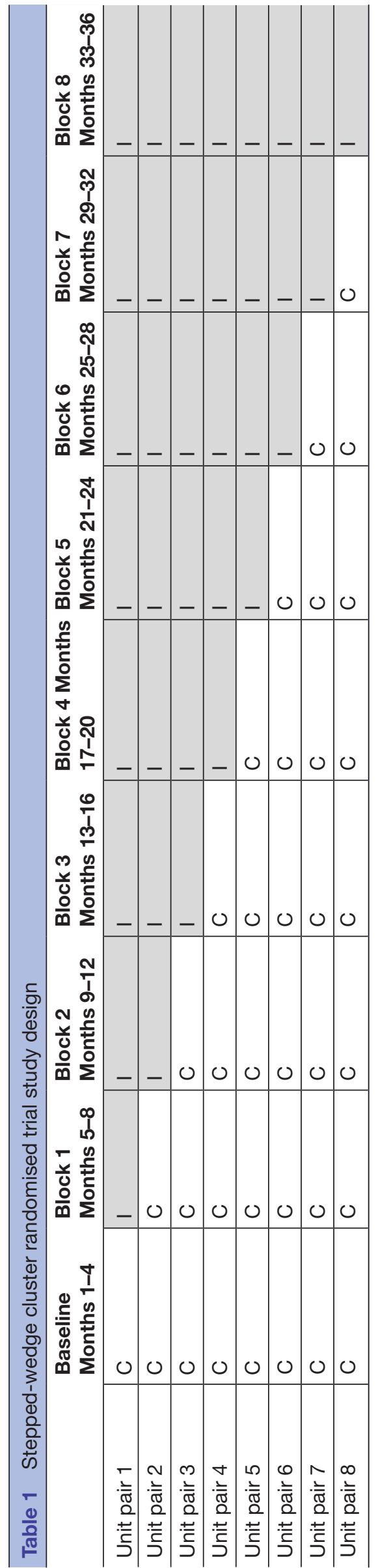

This study has received institutional IRB approval from the University of Iowa and site-specific IRB approval, including waiver of documentation of informed consent. We will offer participants the opportunity to talk with the treating physician or their family member before consenting. Study participation will not influence the standard of care subjects would otherwise receive for their disease process. To minimise risks, all subjects will be carefully prescreened to identify any factors that could contribute to increased risk. We will capture adherence to the intervention during repeated site visits and patient surveys administered throughout the intervention period, and we will record patient drop-out. We will store all confidential information in locked offices and store electronic data on password-protected computers only available to study team members. Participants will receive study team members' contact information.

\section{Sample size and power}

We used the method described by Hussey and Hughes to calculate the sample size and the study's power. ${ }^{27}$ As described above, the stepped-wedge study will last 36 months (time points) with data collected monthly from 16 sites (clusters). After the baseline period, two sites will transition to the intervention at the start of each subsequent 4-month block. Our pilot data suggested that approximately 1825 patients will receive haemodialysis at any given time across all sites, with approximately 100 patients per site per time point $(\mathrm{N})$. We estimated the between site variability as $\tau^{2}=0.01$. Given that $3 \%$ of patients who received haemodialysis at our study sites during 2016 acquired S. aureus BSI, we estimated the within-site variability to be 0.00029 . Thus, we estimated that we will have $98 \%$ power to see a change in the rate of $S$. aureus BSI from $3 \%$ to $2 \%$ (absolute difference $=1 \%$, $\mathrm{OR}=0.66$ ). This difference is more conservative than the difference seen in prior mupirocin decolonisation studies among patients on haemodialysis $(\mathrm{OR}=0.32-0.51) .{ }^{19} \mathrm{Our}$ pilot data indicated that $30 \%$ of patients on haemodialysis at our study sites were dialyzed through central venous catheters, 5\% of whom acquired S. aureus BSI in 2016. Given this information, we estimated that a subset analysis of patients dialyzed through catheters will have $99 \%$ power to identify a decrease in infections from $5 \%$ to $2 \%$ (absolute difference $=3 \%$, OR=0.40).

\section{Randomisation}

We paired dialysis units into 2-unit blocks according to two rules: (1) The dialysis units in a pair were not within the same geographical region and (2) The approximate total monthly unique patients in a given unit block would be approximately 150 patients. Two study team members independently created the dialysis unit blocks and they minimised the variation from the ideal unit block size when their pairings disagreed. After we created the two-unit block pairs, we used the sample function in $\mathrm{R}$ without replacement to randomise the order in which the pairs would enter the intervention phase. Each unit block had the same probability of selection. We stored the final 
Table 2 Schedule of events table for both haemodialysis and healthcare worker visits

\begin{tabular}{|c|c|c|c|c|c|c|c|}
\hline Event & Visit 1 & Visit 2 & Visit 3 & Visit 4 & $\begin{array}{l}\text { Each dialysis } \\
\text { appointment }\end{array}$ & $\begin{array}{l}\text { Every } 6 \\
\text { months* }\end{array}$ & Once \\
\hline $\begin{array}{l}\text { Review of inclusion/exclusion criteria and lab results to } \\
\text { confirm subject eligibility }\end{array}$ & $\mathrm{x}$ & $\mathrm{x}$ & & & & & \\
\hline Testing for Staphylococcus aureus nasal carriage & $\mathrm{X}$ & & & & & $\mathrm{X}$ & \\
\hline Preintervention Survey & $x$ & & & & & & \\
\hline Povidone-iodine administration & & $\mathrm{X}$ & & & $\mathrm{X}$ & & \\
\hline Healthcare worker interview & & & & & & & $\mathrm{X}$ \\
\hline
\end{tabular}

${ }^{*}$ A study member will obtain the nasal swabs during the subject's dialysis sessions. This is in addition to the povidone-iodine administration.

randomisation in a password-protected file. Only the two team members who performed the randomisation and $3 \mathrm{M}$, which must coordinate delivery of the product to the participating sites in accordance with the project timeline, have access to that file. We will notify sites 4 months before their planned intervention start date. The research team is well connected with all sites and will prevent premature implementation of the intervention.

\section{Intervention}

Events and procedures for haemodialysis subjects will occur over four research visits (table 2). Research personnel will visit the dialysis centres four times over the study period.

\section{Visit 1}

Approximately a month before a dialysis unit is scheduled to begin the intervention, a study team member will visit the dialysis unit. During the visit, the study team member will describe the study, obtain verbal informed consent from patients present, and administer the preintervention (control) survey to all patients on haemodialysis who agree to participate. A study team member also will swab participants' noses to identify patients who carry $S$. aureus at baseline.

\section{Visit 2}

When a dialysis unit is scheduled to begin the intervention, study personnel will obtain verbal informed consent from patients present and give each participating patient their first disposable, single-use bottles of PVI, 4 applicators and illustrated instructions for use. Participating patients will apply PVI at each haemodialysis appointment. Patients will be encouraged to apply the PVI to their own noses, but they can also ask a nurse or technician for assistance. Patients will also have the option to apply PVI at home.

\section{Visit 3}

After a dialysis session has begun and after obtaining verbal informed consent from the patient, a study team member will administer the first intervention survey to the patient. This intervention survey will assess acceptability of PVI approximately 1 month after the intervention has started.

\section{Visit 4}

Approximately 5 months after the beginning of the intervention and after obtaining verbal informed consent from the patient, a study team member will administer the second intervention survey. The two intervention surveys will ask the same questions and will be performed in the same manner. The results of the control period survey and the two intervention period surveys for each patient can be linked together.

\section{Staphylococcus aureus point prevalence studies}

Each haemodialysis unit will collect nasal swabs from each participating haemodialysis patient's nose during the baseline period and twice per year over the 3-year study period (total of six times including baseline) we will collect nasal swabs to determine $S$. aureus colonisation status.

\section{HCW interview}

Two members of the research team will conduct semistructured in-person interviews with staff during site visits. The semistructured interviews will include open-ended questions to explore domains including barriers and facilitators to implementing the intervention, provider and patient compliance with PVI decolonisation, and the acceptability and feasibility of PVI decolonisation. Interviews will be audiorecorded with the HCWs' permission and transcribed.

\section{Outcomes and data collection}

Primary outcome

The primary outcome of the study will be $S$. aureus BSI, defined as a $S$. aureus positive blood culture collected in the outpatient setting or within one calendar day after a hospital admission. This outcome is collected every month by dialysis staff or infection prevention staff at each hospital system in accordance with The US Centers for Disease Control and Prevention's National Healthcare Safety Network and the US CMS requirements. These data will be shared with the study team and validated via chart review. 
Table 3 Definitions of secondary outcomes (CDC NHSN definitions)

A positive blood specimen collected in the outpatient setting or within one calendar day after a hospital admission.

$\begin{array}{ll}\text { Bloodstream infection (BSI) } & \text { calendar day after a hospital admission. } \\ \text { Access related bloodstream infection (ARBSI) } & \begin{array}{l}\text { A bloodstream infection with the suspected source reported as the vascular } \\ \text { access or uncertain. }\end{array} \\ \begin{array}{l}\text { Staphylococcus aureus ARBSI } \\ \text { Local access site infection }\end{array} & \begin{array}{l}\text { Pus, redness or increased swelling at the vascular access site when an ARBS } \\ \text { is not present. }\end{array} \\ \text { S aureus local access site infection } & \begin{array}{l}\text { Pus, redness or increased swelling at the vascular access site when an ARBS } \\ \text { is not present but with positive culture for S. aureus. }\end{array} \\ \text { S aureus BSI among intervention participants } & \begin{array}{l}\text { An S. aureus positive blood specimen collected in the outpatient setting or } \\ \text { within one calendar day after a hospital admission from patients participating } \\ \text { in the intervention. }\end{array}\end{array}$

CDC NHSN, Centres for Disease Control and Prevention's National Healthcare Safety Network.

\section{Secondary and additional outcomes}

Definitions of secondary outcomes are presented in table 3 with additional evaluated outcomes presented in table 4 .

During each site's intervention period, a member of the study team will swab each participant's nares during their haemodialysis session after the patient applies PVI to determine if patients are colonised with $S$. aureus after applying PVI and during the at-risk period. S. aureus isolates will be tested for methicillin-susceptibility and the research team will perform pulsed field gel electrophoresis on all nasal isolates and if available, bloodstream isolates to assess whether serial isolates from the same patient are related, whether isolates from different patients in the same dialysis unit are related, and whether nasal isolates and infecting isolates from the same patient are identical. A sample of $S$. aureus isolates will be evaluated using whole genome sequencing. Laboratory testing will occur in a single laboratory using standardised methodology.

\section{Statistical and ethnographic analysis}

Objective 1

The study team will evaluate characteristics of individual patients and the clusters by exposure status (control or intervention) to assess the balance between groups. As most participants will take part in both settings, paired t-tests, McNemar's test and repeated measures analysis of variance will be used as appropriate. The overall proportion of unique patients in the control group who acquire infections compared with the intervention group will be assessed via McNemar's Test.

In the primary analysis, the study team will use a generalised linear mixed model (GLMM) with a logit link function to perform an analysis at the individual patient level evaluating the association between nasal PVI and $S$. aureus BSI. The model will include step and intervention indicators as fixed effects and a random intercept for cluster to account for hospital dependence. The study team will statistically adjust for important confounding variables, such as dialysis access type. The study team will perform an intention-to-treat analysis, assuming all patients received nasal PVI during the intervention periods. Patients who stop using PVI will be included in the study and evaluated for outcomes in this intention-to-treat analysis. Reasons for study 'drop-out' such as death or renal transplant are not related to the intervention. However, the study team will model time to drop-out to characterise this patient population. An analysis will also be performed among patients who met the inclusion criteria, agreed to participate in the study, and received at least one dose of PVI.

Because patients who receive haemodialysis through central venous catheters are at the highest risk of infection, the study team use the GLMM methods described above to evaluate the association between nasal PVI and S. aureus BSI stratified by dialysis access type. The study team will also perform exploratory analyses to assess the effect of rural vs urban haemodialysis units and the effect of nasal PVI on methicillin-resistant $S$. aureus and

Table 4 Additional outcomes evaluated

Patient satisfaction with nasal PVI

Healthcare worker satisfaction with intervention

Staphylococcus aureus colonisation
Barriers and facilitators to the intervention collected from patients through qualitative surveys.

Barriers and facilitators to the intervention collected through qualitative interviews with healthcare workers.

The presence of $S$. aureus in the nares.

$\mathrm{PVI}$, povidone-iodine. 
methicillin-susceptible $S$. aureus BSIs separately. Finally, the study team will evaluate the effect of nasal PVI on the secondary outcomes: all BSI caused by any pathogen, all BSI caused by any pathogen, local access site infection and vascular access infection. The study team will use SAS V.9.4 for all analyses.

\section{Objective 2}

The study team will calculate descriptive statistics for control and intervention survey questions. They will use a two-sample Fisher's exact test to assess differences in the patients' responses to specific questions during the control and intervention periods. The study team will use bivariable and multivariate regression analysis to explore associations between survey measures and covariates.

\section{Objective 3}

Investigators will read a subset of transcripts and generate a preliminary codebook using an integrated approach to thematic analysis that includes a priori project-specific thematic codes, Consolidated Framework for Implementation Research constructs and inductive codes identified during team discussions. Thereafter, the team will code documents, then iteratively adapt the codebook, conduct preliminary analyses, adapt the interview guide if needed and gauge whether data saturation (ie, no new themes or patterns emerge) has been reached. If data saturation has not been attained or if new areas are identified, we will perform, record and analyse additional telephone interviews. The team will document codebook changes and the rationale for each change and will keep an audit trail.

\section{Patient and public involvement}

Neither patients nor members of the public participated in designing this study.

\section{Ethics and dissemination}

The risk to patients is low in this study; however, a data and safety monitoring board (DSMB) will oversee this study. The DSMB will be made up of clinical, biostatistical, infectious disease and renal disease experts who are approved by the Agency for Health Research and Quality. Occurrence of adverse events will be monitored throughout the trial by surveys and the study team, and will cover all randomised subjects. Rare allergy to PVI will be treated by haemodialysis staff if needed. This is a phase IV study of an antiseptic that is available under the FDA Final Rule (Federal Register December 20, 2017). ${ }^{25}$ Any potential side effects from PVI will be captured through patient surveys. To protect confidentiality, we will assign each subject a study ID. All electronic files are stored on password-protected computers that are connected to a secured shared drive. Nasal swabs will be labelled with a coding descriptor, and no PHI will be collected from the lab. The isolates will be discarded after the results are finalised. Only the PI, data analysts, statistician and the DSMB will have access to the final trial data set. Site principal investigators will have direct access to their own site's data sets, and will have access to other sites' data by request. In year 5 of the study, we will present our results at international meetings. We will publish our findings in peer-reviewed journals and make each peer-reviewed accepted manuscript publicly available.

\section{DISCUSSION}

Prior studies have found that nasal decolonisation with mupirocin reduced infection rates among patients on haemodialysis. ${ }^{131619}$ For example, Weiner et al demonstrated that nasal mupirocin was associated with a fourfold reduction in $S$. aureus BSIs in this patient population. ${ }^{28}$ However, consistent use of mupirocin can lead to mupirocin-resistant $S$. aureus. ${ }^{13}$ A meta-analysis found that decolonisation with mupirocin was associated with a 59\% reduction in S. aureus infections among dialysis patients, but up to $10 \%$ of patients who used mupirocin become colonised with a mupirocin-resistant $S$. aureus strain. ${ }^{19}$ Given that mupirocin prophylaxis can increase the frequency of mupirocin-resistant $S$. aureus isolates, and that the mupirocin decolonisation protocol is often difficult to implement, most haemodialysis units do not routinely decolonise patients with mupirocin. ${ }^{19}$

Nasal PVI may be preferred over mupirocin for longterm prevention of $S$. aureus infections because it is easy to use and it has multiple targets of action-thus, the risk of PVI resistance among $S$. aureus isolates is minimal. ${ }^{23}$ 29-31 PVI has been used in healthcare for years for skin antisepsis. Recently, small, single-centre studies found that nasal PVI was associated with decreased surgical site infection rates, and that surgical patients preferred this product over mupirocin because it had fewer side effects and was more pleasant. ${ }^{32-35}$ Some investigators have used PVI at haemodialysis catheter exit sites or for catheter care. ${ }^{36}{ }^{37}$ However, no published studies have evaluated nasal PVI for decolonising patients on haemodialysis.

Our objectives are to evaluate whether decolonising patients' noses with PVI will reduce rates of S. aureus BSI among patients on haemodialysis, to qualitatively evaluate the implementation of this intervention, and to assess patient and HCW satisfaction with PVI. This trial will be performed at 16 outpatient haemodialysis units affiliated with five academic medical centres. These ambulatory haemodialysis units are geographically dispersed and care for both rural and urban patients who receive chronic care.

We chose the stepped-wedge CRT design for multiple reasons. First, since nasal PVI could prevent endogenous $S$. aureus infection and could prevent exogenous transmission of this organism from patient to patient, individual randomisation would not allow us to adequately assess the full effect of this intervention. Second, units will serve as their own controls and as controls for other units, thus limiting selection bias and imbalance among the intervention and control units. ${ }^{26}$ Third, the staggered starting dates can help us measure and adjust for temporal biases such as the effect of CMS policy changes that occur during the study period. 


\section{Limitations}

The proposed study has three main limitations. First, nasal PVI suppresses bacteria for only 12-24 hours. ${ }^{31}$ Thus, PVI must be reapplied before each procedure. Second, we will not compare PVI with mupirocin. Instead, our control group will be standard care, which is justified because mupirocin has not been routinely used for preventing BSI among patients on haemodialysis due to implementation barriers. Third, PVI is considered a novel intervention for patients on haemodialysis, and thus, we are required to obtain informed consent from each patient. Therefore, patients who do not consent to using nasal PVI could transmit $S$. aureus to patients who do participate in the intervention.

\section{Significance}

Nasal PVI is currently used in many hospitals to prevent surgical site infections. Our study evaluates this product in a new patient population. This large stepped-wedge CRT aims to determine whether nasal PVI decreases rates of $S$. aureus BSI among patients on haemodialysis, and to collect data on barriers and facilitators to implementation. Given that PVI as widely available and inexpensive, is easy to use and implement, and does not cause resistance, this intervention could be more generalisable than mupirocin ointment. An effective intervention to prevent infections among patients on haemodialysis could improve outcomes among the 2 million people who receive renal replacement therapy worldwide. ${ }^{38}$

\section{Trial status}

Trial is currently ongoing.

\section{Author affiliations}

${ }^{1}$ Internal Medicine, The University of lowa Roy J and Lucille A Carver College of Medicine, lowa City, lowa, USA

${ }^{2}$ Renal Medicine, Emory University School of Medicine, Atlanta, Georgia, USA

${ }^{3}$ Emory Antibiotic Resistance Center, Emory University School of Medicine, Atlanta, Georgia, USA

${ }^{4}$ Department of Medicine, University of Pennsylvania Perelman School of Medicine, Philadelphia, Pennsylvania, USA

${ }^{5}$ Division of Infectious Diseases, University of Illinois College of Medicine, Chicago, Illinois, USA

${ }^{6}$ Division of Nephrology, Washington University School of Medicine in Saint Louis, St Louis, Missouri, USA

${ }^{7}$ Clinical Research Unit, The University of lowa Hospitals and Clinics, lowa City, lowa, USA

${ }^{8}$ Biostatistics, Epidemiology, and Informatics, University of Pennsylvania Perelman

School of Medicine, Philadelphia, Pennsylvania, USA

${ }^{9}$ Division of Infectious Diseases, Emory University School of Medicine, Atlanta,

Georgia, USA

${ }^{10}$ Department of Medicine, University of Illinois College of Medicine, Chicago, Illinois, USA

Contributors Study design: MLS, RN, KD, LAH, AMJO'S, LB, DD, JC, JJ, DP, SB, AV, AM, MF and DO-M. Study implementation: MLS, MAW, RN, KD, A-MR, LB, JC, JJ, DP, SB, AV, AM, MF, PT, MM and EJ. Statistical analysis: RN and AMJO'S. All authors contributed to, read and approved the final manuscript. MLS had full access to all of the data in the study and takes responsibility for the integrity of the data and the accuracy of the data analysis.

Funding This work was supported by the Agency for Health Research and Quality (AHRQ) grant number 1R01HS026724-01 and by the National Center For Advancing Translational Sciences of the National Institutes of Health under Award Number
UL1TR002537. Povidone-iodine product will be donated by 3M (funding number ISR74).

Disclaimer $\mathrm{AHRQ}$, NIH and 3M have no role in study design; collection, management, analysis, and interpretation of data; writing of the report; nor the decision to submit the report for publication.

Competing interests None declared.

Patient and public involvement Patients and/or the public were not involved in the design, or conduct, or reporting, or dissemination plans of this research.

Patient consent for publication Not applicable.

Provenance and peer review Not commissioned; externally peer reviewed.

Open access This is an open access article distributed in accordance with the Creative Commons Attribution Non Commercial (CC BY-NC 4.0) license, which permits others to distribute, remix, adapt, build upon this work non-commercially, and license their derivative works on different terms, provided the original work is properly cited, appropriate credit is given, any changes made indicated, and the use is non-commercial. See: http://creativecommons.org/licenses/by-nc/4.0/.

ORCID iD

Ana-Monica Racila http://orcid.org/0000-0002-5751-5422

\section{REFERENCES}

1 United States Renal Data System. USRDS annual data report: epidemiology of kidney disease in the United States. Bethesda, MD: National Institutes of Health, National Institute of Diabetes and Digestive and Kidney Diseases (US), 2020.

2 Dalrymple LS, Mu Y, Romano PS, et al. Outcomes of infectionrelated hospitalization in Medicare beneficiaries receiving in-center hemodialysis. Am J Kidney Dis 2015;65:754-62.

3 Patel PR, Kallen AJ, Arduino MJ. Epidemiology, surveillance, and prevention of bloodstream infections in hemodialysis patients. Am J Kidney Dis 2010;56:566-77.

4 Nguyen DB, Shugart A, Lines C, et al. National healthcare safety network (NHSN) dialysis event surveillance report for 2014. Clin J Am Soc Nephrol 2017;12:1139-46.

5 Landry DL, Braden GL, Gobeille SL, et al. Emergence of gentamicinresistant bacteremia in hemodialysis patients receiving gentamicin lock catheter prophylaxis. Clin J Am Soc Nephrol 2010;5:1799-804.

6 Maki DG, Ash SR, Winger RK, et al. A novel antimicrobial and antithrombotic lock solution for hemodialysis catheters: a multicenter, controlled, randomized trial. Crit Care Med 2011;39:613-20.

7 Engemann JJ, Friedman JY, Reed SD, et al. Clinical outcomes and costs due to Staphylococcus aureus bacteremia among patients receiving long-term hemodialysis. Infect Control Hosp Epidemiol 2005;26:534-9.

8 Nissenson AR, Dylan ML, Griffiths RI, et al. Clinical and economic outcomes of Staphylococcus aureus septicemia in ESRD patients receiving hemodialysis. Am J Kidney Dis 2005;46:301-8.

$9 \mathrm{Li} \mathrm{Y,} \mathrm{Friedman} \mathrm{JY,} \mathrm{O'Neal} \mathrm{BF,} \mathrm{et} \mathrm{al.} \mathrm{Outcomes} \mathrm{of} \mathrm{Staphylococcus}$ aureus infection in hemodialysis-dependent patients. Clin J Am Soc Nephrol 2009;4:428-34.

10 Saxena AK, Panhotra BR, Venkateshappa CK, et al. The impact of nasal carriage of methicillin-resistant and methicillin-susceptible Staphylococcus a ureus (MRSA \& MSSA) on vascular access-related septicemia among patients with type-II diabetes on dialysis. Ren Fail 2002;24:763-77.

11 Vaziri ND, Pahl MV, Crum A, et al. Effect of uremia on structure and function of immune system. J Ren Nutr 2012;22:149-56.

12 Nguyen DB, Lessa FC, Belflower R, et al. Invasive methicillinresistant Staphylococcus aureus infections among patients on chronic dialysis in the United States, 2005-2011. Clin Infect Dis 2013;57:1393-400.

13 Boelaert JR, Van Landuyt HW, Godard CA, et al. Nasal mupirocin ointment decreases the incidence of Staphylococcus aureus bacteraemias in haemodialysis patients. Nephrol Dial Transplant 1993;8:235-9.

14 Schmid H, Romanos A, Schiffl H, et al. Persistent nasal methicillinresistant Staphylococcus aureus carriage in hemodialysis outpatients: a predictor of worse outcome. BMC Nephrol 2013;14:93.

15 Yu VL, Goetz A, Wagener M, et al. Staphylococcus aureus nasal carriage and infection in patients on hemodialysis. Efficacy of antibiotic prophylaxis. N Engl J Med 1986;315:91-6.

16 Boelaert JR, Van Landuyt HW, Gordts BZ, et al. Nasal and cutaneous carriage of Staphylococcus aureus in hemodialysis patients: 
the effect of nasal mupirocin. Infect Control Hosp Epidemiol 1996;17:809-11.

17 Grothe C, Taminato M, Belasco A, et al. Prophylactic treatment of chronic renal disease in patients undergoing peritoneal dialysis and colonized by Staphylococcus aureus: a systematic review and metaanalysis. BMC Nephrol 2016;17:115.

18 Dalrymple LS, Johansen KL, Chertow GM, et al. Infection-Related hospitalizations in older patients with ESRD. Am J Kidney Dis 2010;56:522-30.

19 Nair R, Perencevich EN, Blevins AE, et al. Clinical Effectiveness of Mupirocin for Preventing Staphylococcus aureus Infections in Nonsurgical Settings: A Meta-analysis. Clin Infect Dis 2016;62:618-30.

20 Pérez-Fontán M, Rosales M, Rodríguez-Carmona A, et al. Mupirocin resistance after long-term use for Staphylococcus aureus colonization in patients undergoing chronic peritoneal dialysis. Am J Kidney Dis 2002;39:337-41.

21 Tacconelli E, Carmeli Y, Aizer A, et al. Mupirocin prophylaxis to prevent Staphylococcus aureus infection in patients undergoing dialysis: a meta-analysis. Clin Infect Dis 2003;37:1629-38.

22 Kluytmans JA, Manders MJ, van Bommel E, et al. Elimination of nasal carriage of Staphylococcus aureus in hemodialysis patients. Infect Control Hosp Epidemiol 1996;17:793-7.

23 Kunisada T, Yamada K, Oda S, et al. Investigation on the efficacy of povidone-iodine against antiseptic-resistant species. Dermatology 1997;195 Suppl 2:14-18.

24 Barry AL, Fuchs PC, Brown SD. Lack of effect of antibiotic resistance on susceptibility of microorganisms to chlorhexidine gluconate or povidone iodine. Eur J Clin Microbiol Infect Dis 1999;18:920-1.

25 Food and Drug Administration, HHS.. Safety and effectiveness of health care antiseptics; topical antimicrobial drug products for overthe-counter human use. final rule. Fed Regist 2017;82:60474.

26 Hemming K, Haines TP, Chilton PJ, et al. The stepped wedge cluster randomised trial: rationale, design, analysis, and reporting. BMJ 2015;350:h391.

27 Hussey MA, Hughes JP. Design and analysis of stepped wedge cluster randomized trials. Contemp Clin Trials 2007;28:182-91.
28 Weiner LM, Webb AK, Limbago B, et al. Antimicrobial-Resistant pathogens associated with healthcare-associated infections: summary of data reported to the National healthcare safety network at the centers for disease control and prevention, 2011-2014. Infection Control \& Hospital Epidemiology 2016;37:1288-301.

29 Lanker Klossner B, Widmer H-R, Frey F. Nondevelopment of resistance by bacteria during Hospital use of Povidone-lodine. Dermatology 1997;195:10-13.

30 Gocke DJ, Ponticas S, Pollack W. In vitro studies of the killing of clinical isolates by povidone-iodine solutions. J Hosp Infect 1985;6 Suppl A:59-66.

31 Anderson MJ, David ML, Scholz M, et al. Efficacy of skin and nasal povidone-iodine preparation against mupirocin-resistant methicillinresistant Staphylococcus aureus and $S$. aureus within the anterior nares. Antimicrob Agents Chemother 2015;59:2765-73.

32 Torres EG, Lindmair-Snell JM, Langan JW, et al. Is Preoperative Nasal Povidone-lodine as Efficient and Cost-Effective as Standard Methicillin-Resistant Staphylococcus aureus Screening Protocol in Total Joint Arthroplasty? J Arthroplasty 2016;31:215-8.

33 Maslow J, Hutzler L, Cuff G, et al. Patient experience with mupirocin or povidone-iodine nasal decolonization. Orthopedics 2014;37:e576-81.

34 Phillips M, Rosenberg A, Shopsin B, et al. Preventing surgical site infections: a randomized, open-label trial of nasal mupirocin ointment and nasal povidone-iodine solution. Infect Control Hosp Epidemiol 2014;35:826-32

35 Bebko SP, Green DM, Awad SS. Effect of a preoperative decontamination protocol on surgical site infections in patients undergoing elective orthopedic surgery with hardware implantation. JAMA Surg 2015;150:390.

36 Fong IW. Prevention of haemodialysis and peritoneal dialysis catheter related infection by topical povidone-iodine. Postgrad Med J 1993;69 Suppl 3:515-7.

37 Al-Said J, Pagaduan AC. Infection-free hemodialysis: can it be achieved? Saudi J Kidney Dis Transp/ 2009;20:677.

38 Liyanage T, Ninomiya T, Jha V, et al. Worldwide access to treatment for end-stage kidney disease: a systematic review. Lancet 2015;385:385. 